\title{
Prototipo de videolaringoscopio: Wi-Mac-Multivision
}

\author{
Prototype of videolaringoscope: Wi-Mac-;Multivision
}

Jonathan De Freitas H. ${ }^{1}$, Javier E. Moreno S. ${ }^{1}$, Miguel Silva ${ }^{1}$

\begin{abstract}
Introduction: The management of the airway is a constant challenge for anesthesiologists, there are devices that facilitate orotracheal intubation, among which videolaryngoscopes can be mentioned, in view of their high costs it is difficult to access them. The following study is a prototype of a low cost and highly in the Central Hospital of Maracay (HCM). Materials and Methods: Non-experimental, prospective and observational design, under the modality of a special project with an evaluative scope. The population consists of 32 patients who required orotracheal intubation by the anesthesiology and resuscitation service of the HCM with a video-laryngoscope prototype from a Macintosh laryngoscope. Results: The use of Wi-Mac-Multivision showed $100 \%$ of orotracheal intubations without presenting any type of complication in them, only one of the patients deserved a second attempt to achieve orotracheal intubation. It was possible to visualize the vocal cords according to the classification of Cormack-Lehane in CL-I $93.7 \%$ and CL-II $6.3 \%$. In only 3 cases $(9.4 \%)$ was it necessary to use external maneuvers that improved visualization of the vocal cords (Maneuvers of B.U.R.P.) The average time needed to achieve orotracheal intubation was $29.62 \pm 7.53$ seconds. Conclusions: WiMac-Multivision manages to prove to be an alternative to be used by the staff of the central hospital to achieve orotracheal intubations, its low cost makes it easy to replicate this device and benefit more patients. Such a device would have advantages over the learning and training of HCM personnel. It remains to evaluate the number of intubations to achieve a good degree of training, indications and if it can be used in difficult airway patients, that is why there is a line of research open in our institution, which is expected to continue.
\end{abstract}

Servicio de Anestesiología y Reanimación, Hospital Central de Maracay. Venezuela, Edo. Aragua.

Fecha de recepción: 08 de agosto de 2019

Fecha de aceptación: 28 de septiembre de 2019

\section{ORCID}

https://orcid.org/0000-0002-6357-5448

Correspondencia:

Dr. Jonathan De Freitas H

Email: homenjonathan89@hotmail.com 


\section{RESUMEN}

Introducción: El manejo de la vía aérea es un reto constante para los anestesiólogos, existen dispositivos que facilitan la intubación orotraqueal entre los cuales se pueden mencionar los videolaringoscopios, en vista de sus altos costos resulta difícil acceder a ellos. El siguiente estudio se trata de un prototipo de videolaringoscopio de bajo costo y alta efectividad que busca solventar un vacío en dispositivos para el manejo de la vía aérea en el Hospital Central de Maracay (HCM). Materiales y Métodos: Diseño no experimental, prospectivo y observacional, bajo la modalidad de proyecto especial y con alcance evaluativo. La población consta de 32 pacientes que ameritaron intubación orotraqueal por parte del Servicio de Anestesiología y Reanimación del HCM con un prototipo de videolaringoscopio a partir de un laringoscopio de Macintosh. Resultados: El uso del Wi-Mac-Multivision arrojó un 100\% de intubaciones orotraqueales sin presentar ningún tipo de complicación en ellas, solo uno de los pacientes ameritó un segundo intento para lograr la intubación orotraqueal. Se logró visualizar las cuerdas vocales según la clasificación de Cormack-Lehane en CL-I $93,7 \%$ y CL-II 6,3\%. En solo 3 casos (9,4\%) fue necesario el empleo de maniobras externas que mejoraron la visualización de las cuerdas vocales (maniobras de B.U.R.P.). El tiempo medio necesario para lograr la intubación orotraqueal fue de 29,62 \pm 7,53 segundos. Conclusiones: Wi-Mac-Multivision logra demostrar ser una alternativa a ser empleada por el personal del hospital central para lograr intubaciones orotraqueales, su bajo costo hace fácil replicar dicho dispositivo y beneficiar a más pacientes. Dicho dispositivo tendría ventajas sobre el aprendizaje y capacitación del personal del HCM. Queda por evaluar el número de intubaciones para poder lograr un buen grado de entrenamiento, indicaciones y si puede ser empleado en pacientes vías aéreas difícil, es por ello que queda una línea de investigación abierta en nuestra institución, la cual se espera pueda continuar.

\section{Palabras clave:}

Intubación, tecnología, videolaringoscopio

\section{Introducción}

- I manejo de la vía aérea es un reto constante para Eel médico y muy particularmente para el anestesiólogo ya que, en su quehacer, diario comúnmente se enfrenta a situaciones en las cuales puede tener dificultades para acceder a ésta y lograr la intubación endotraqueal del paciente.

La intubación endotraqueal sigue siendo la medida más efectiva para asegurar una vía aérea permeable, la cual requiere experiencia para realizar dicho procedimiento. Aproximadamente, el $30 \%$ de todas las muertes atribuibles a la anestesia es debido a la dificultad para manejar la vía aérea[1].

La vía aérea difícil es la situación clínica en el cual el personal con entrenamiento convencional experimenta dificultad para la ventilación de la vía aérea superior con mascarilla facial, o dificultad para la intubación orotraqueal o ambas[2].

Frecuentemente, los hallazgos físicos en la explo- ración de la vía aérea discriminan mal entre intubaciones potencialmente fáciles y difíciles, por lo que se debe estar preparado siempre para abordar una vía aérea difícil no predicha, ya que muchos de estos pacientes han tenido un examen físico considerado como "tranquilizador"[3],[4].

Además, la intubación emergente fuera del quirófano se asocia con un riesgo mucho mayor de laringoscopia difícil. De tal manera que las técnicas que pueden mejorar la intubación exitosa pueden ser especialmente útiles en estos entornos[5].

El anestesiólogo inglés Sir Robert Reynolds Macintosh presentó en 1943 el laringoscopio con hoja curva, la cual permite una mejor visión de las cuerdas vocales y en consecuencia facilita la intubación endotraqueal[6]; es desde ese momento que la laringoscopia directa ha constituido para los anestesiólogos el Gold standard para la intubación orotraqueal.

Ocasionalmente, la laringoscopia directa produce una pobre visualización laríngea, lo que hace más 
probable que se requieran múltiples y prolongados intentos de intubación los cuales pueden o no estar asociados a complicaciones como desaturación de oxígeno, lesión dental y de la vía aérea, daño neurológico e incluso la muerte[7].

El desarrollo de los videolaringoscopios representa el mayor avance en el manejo de la vía aérea en estas últimas décadas, el cual es un dispositivo que utiliza una cámara de video para visualizar las estructuras de la vía aérea. La videolaringoscopia permite aprender de forma sencilla las técnicas de manejo de la vía aérea al visualizar la glotis sin necesidad de obtener una línea directa[7].

El hecho de que los VDL posean el sensor de imagen en la parte distal de la hoja hace que se tenga una visión panorámica de la glotis sin necesidad de "alinear los ejes". Los VDL tienen un campo visual entre $45^{\circ}$ y $60^{\circ}$ a diferencia de la visión distante y tubular de $15^{\circ}$ que proporciona una laringoscopia clásica[8],[9].

La videolaringoscopia, comparada con la laringoscopia directa para intubaciones difíciles, proporciona una visión significativamente mejor de las cuerdas vocales, una mayor tasa de éxito, intubaciones más rápidas y menos necesidad de optimizar las maniobras. Por lo tanto, se considera que la videolaringoscopia conduce a una mejora clínicamente relevante de las condiciones de intubación y puede recomendarse para el manejo de las vías aéreas difíciles[10]

A partir de 2013 la Sociedad Americana de Anestesiología (ASA) incluye en su algoritmo de vía aérea difícil la utilización de VDL, proponen usarlos cuando la ventilación con mascarilla facial es efectiva y se ha realizado un intento previo de intubación con laringoscopio directo. En dicho algoritmo fueron mencionados de forma genérica varios modelos de VDL, sin especificar ni valorar cuál instrumento se debe emplear[2].

De igual manera la Difficult Airway Society (DAS) en la última actualización de sus guías en 2015 para el manejo de vías aéreas difíciles no anticipadas se puede observar que los VDL entran por fin en el Plan A, en el abordaje rutinario de la vía aérea[11].

Las publicaciones sobre VDL demuestran la eficacia de estos dispositivos ópticos en situaciones de vía aérea difícil prevista y en los obesos mórbidos, donde al compararlos con el laringoscopio estándar Macintosh se han demostrado más fáciles de utilizar para los principiantes, además de útiles en la docencia de la vía Aérea, ya que el monitor comparte la visión obtenida por el alumno[12].

Los VDL son dispositivos sumamente costosos[13] y de difícil acceso, lo cual es una limitante en nuestro país y en muchos países en vías de desarrollo, por lo que no están disponibles en nuestros centros hospitalarios.

Sin embargo, a pesar de lo mencionado previamente, el doctor Grünberg en el Hospital de Clínicas en Montevideo en el año 2012, ante la necesidad de resolver una vía aérea difícil que requería intubación nasotraqueal para cirugía maxilofacial, utilizó un "videolaringoscopio artesanal" para el cual modificó un laringoscopio convencional agregando una mini cámara fijada con adhesivo a la hoja Macintosh la cual reproducía bajo visión indirecta en una pantalla[14].

En 2016, el anestesiólogo Velásquez-Murillo en México, pudo elaborar con 20 USD un videolaringoscopio artesanal de gran utilidad en la práctica en una serie de casos de vías aéreas difíciles en diferentes escenarios clínicos cotidianos, empleando una cámara endoscópica, que permitiera utilizar monitores (móviles o no móviles), sencilla de limpiar y reutilizable. Adaptando dicha cámara con un broche de acero inoxidable templado el cual se ajustó a la hoja convencional para que la visión estuviera alineada sin partes móviles que pudiesen desprenderse durante la exploración[1].

En 2015, estudiantes del postgrado de anestesiología del Hospital Dr. Luis Razetti de Barinas, diseñaron el primer videolaringoscopio artesanal de efectividad comprobada en Venezuela el cual se encuentra en funcionamiento para el beneficio de la población local, el cual tuvo un bajo costo y por ser un dispositivo liviano es de fácil desplazamiento[15].

Actualmente, en nuestros recintos de salud contamos con el dispositivo por excelencia para el manejo de la vía aérea, que como bien se dijo anteriormente es el laringoscopio de visión directa de Macintosh y un fibrobroncoscopio de un solo uso marca Ambu.

Basándonos en los antecedentes previamente mencionados y ante la constante necesidad de adquirir nuevas alternativas, conocimientos y habilidades para asegurar la vía aérea, surge la inquietud de contar con un VDL haciendo uso de los recursos disponibles con la finalidad de resolver problemas frecuentes y a veces inesperados de la vía aérea en el Servicio de Anestesiología del Hospital Central de Maracay (HCM), de lo cual parte la siguiente interrogante: ¿Existe realmente la necesidad de contar con un videolaringoscopio en el servicio de anestesiología y Reanimación del Hospital Central de Maracay? ¿Es posible mejorar las propuestas anteriores de videolaringoscopios artesanales? ¿Qué elementos son necesarios para dicha modificación? ¿Es factible la realización de dicha propuesta? 


\section{Materiales y Métodos}

El estudio se realizó entre el período abril a noviembre de 2017, y la investigación se realizó en el área de quirófano del Hospital Central de Maracay.

Para la realización del diagnóstico acerca de la necesidad de contar con un videolaringoscopio en el Servicio de Anestesiología y Reanimación, se procedió a realizar un cuestionario a la mayor parte de los miembros integrantes del Servicio de Anestesiología y Reanimación donde estuvieron incluidos tantos los médicos residentes (24) y especialistas en anestesiología (25) en el cual se procederá a conocer la opinión de cada integrante a fin de conocer la existencia o no de la necesidad.

Una vez realizado el diagnóstico se procedió con el diseño, para el cual se necesitó como elemento básico un laringoscopio de Macintosh con hojas de diferentes tamaños (3 y 4). Adicionalmente, una cámara endoscópica (costo aproximado: 30 USD), la cual es resistente al agua, con un diámetro de $7 \mathrm{~mm}$, con luz fría propia ( 6 bombillos Leds), sencilla de limpiar de secreciones lo cual permite su reutilización y desinfección, adicionalmente tiene un ángulo de visión de $62^{\circ}$ y tiene un alcance de transmisión de imagen de 15 metros vía Wi-Fi hasta para máximo 4 dispositivos de manera simultánea. Dicha cámara se fijó con dos láminas (broches) de aluminio moldeable justo al lado de la fuente luminosa del laringoscopio y 2 $\mathrm{cm}$ sujetando el cable, aportando así mejor firmeza y acoplamiento (Figuras 1 y 2), con una angulación que permita visualizar la punta del laringoscopio y que esté alineado con las estructuras anatómicas para mejor referencia al momento de realizar la intubación orotraqueal. Dicha cámara amerita corriente eléctrica para su funcionamiento, motivo por el cual se emplea mediante cable USB una conexión a una batería portátil (Powerbank) la cual suministra dicha fuente eléctrica y permite su uso.

Se ameritó un medio donde reproducir las imágenes capturadas por la cámara, por lo que se necesitó un Smartphone con tecnología Wi-Fi que bien cuente con sistema operativo Android o IOS. Adicional de esto dicha cámara incluye una aplicación gratuita para ambos sistemas operativos mencionados anteriormente llamada WIFI_EYE la cual permite el enlace entre la cámara endoscopio y el/los smarthphone(s) conectado(s).

El videolaringoscopio fue manipulado por un solo operador al momento de realizar la intubación orotraqueal, y por lo que resultó importante contar con un medio de fijación para el Smarthphone que reproducirá la imagen de manera que se utilizó una base de

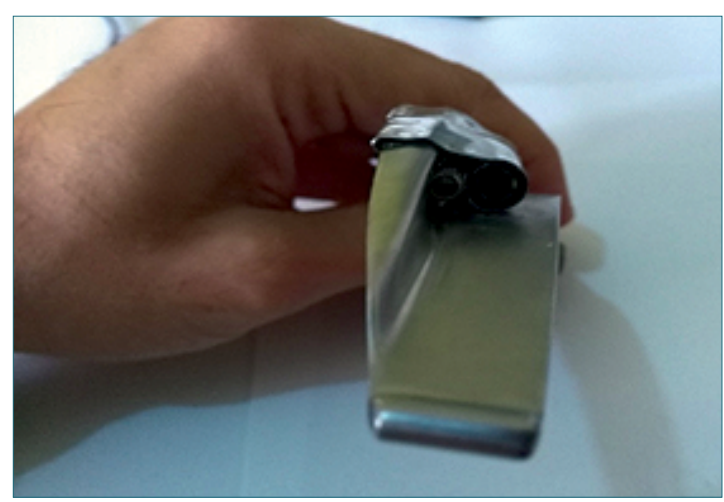

Figura 1. Vista frontal de hoja Macintosh $\mathrm{N}^{\circ} 3$ con cámara sujetada por broche anterior.

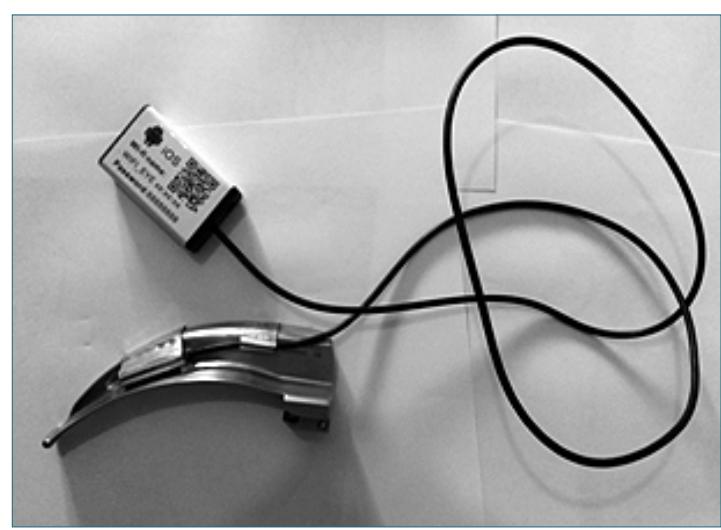

Figura 2. Vista lateral de hoja Macintosh $\mathrm{N}^{\circ} 3$ con cámara sujetada por broche anterior y posterior.

fijación utilizada para anclar teléfonos a manubrios de bicicleta la cual se adaptará al extremo del mango del laringoscopio, dicha base gira en $360^{\circ}$ lo que permite colocar el teléfono en distintos ángulos de inclinación de manera que sea más cómoda la visualización por parte del operador (Figura 3).

Una vez diseñado el videolaringoscopio (Figura 4) el siguiente paso fue la aplicación, el cual será empleado por el investigador de la siguiente manera: Previa oxigenación al 100\% durante tres minutos, se procede a inducción anestésica vía endovenosa, se procederá a aplicar prueba de ventilación la cual debe ser positiva para la aplicación del videolaringoscopio, una vez pasado el tiempo de inicio de acción de los fármacos empleados y con el paciente en buen plano anestésico se procederá con la videolaringoscopia, de manera tal que se coloca la cabeza del paciente en posición de "olfateo", se realiza apertura bucal, 


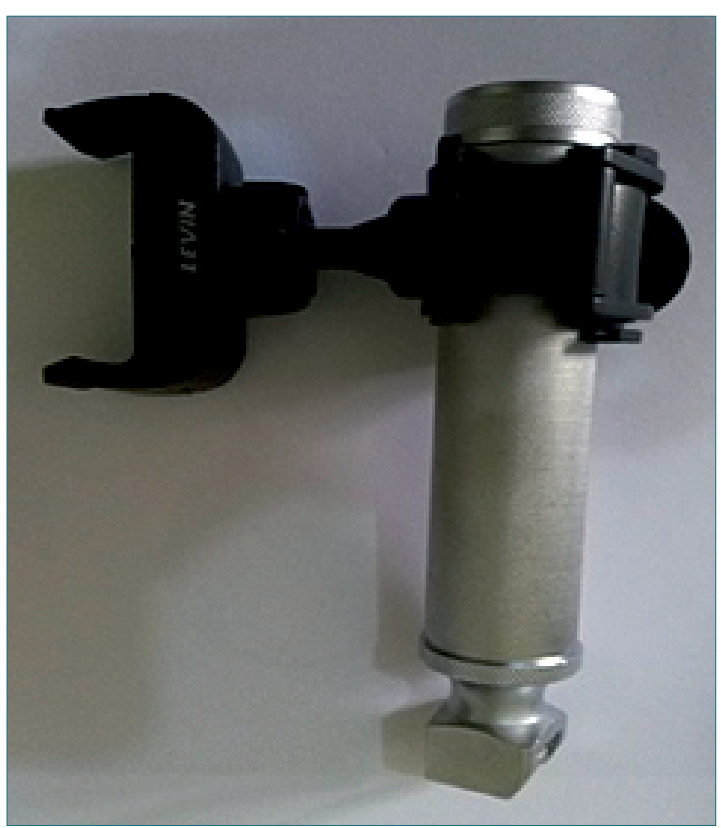

Figura 3. Base de manubrio de bicicleta adaptado para fijar smartphone a mango de laringoscopio.

y se introduce la hoja Macintosh por línea media, se descenderá por la base de la lengua observando las estructuras anatómicas en todo momento por el/los smartphones, ubicando la epiglotis y posicionando la punta de la hoja Macintosh en la vallécula y realizando una ligera fuerza sobre el mango del laringoscopio hacia arriba y hacia los pies del paciente, logrando de este modo la visualización de las cuerdas vocales.

Una vez visualizada la glotis se procedió a introducir el tubo endotraqueal el cual para facilitar la intubación se introdujo con un guiador o estilete con una angulación de hasta $90^{\circ}$. La introducción del tubo se debe seguir bajo visión indirecta en el/los smartphone(s) de manera tal que una vez la punta de éste pase a través de las cuerdas vocales se proceda a retirar el guiador y proseguir con la introducción del tubo hasta que el balón pase por completo las cuerdas vocales (Figuras 5 y 6 ).

La población objeto del estudio fue de 32 pacientes que ingresaron en el Servicio de Anestesiología y Reanimación del Hospital Central de Maracay que ameritaron intubación orotraqueal. Se eligieron pacientes programados para cirugía general de electiva y emergencia con una edad mínima de 18 años, estados físicos ASA I, II, y III de acuerdo con la clasificación de la Sociedad Norteamericana de Anestesiología y quienes pudieron ser ventilados por mascara facial. Se

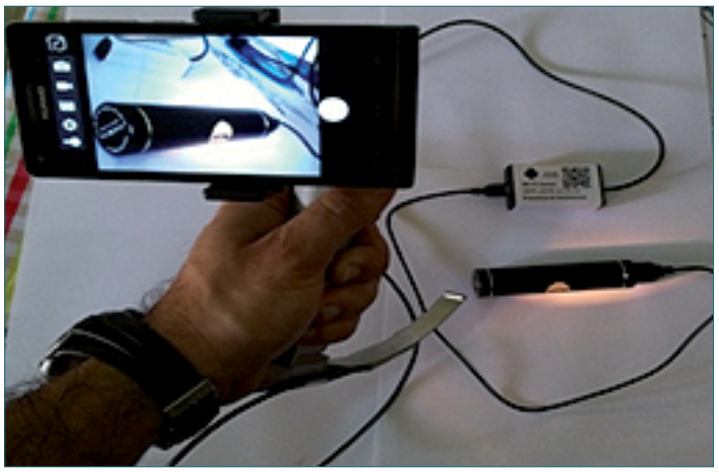

Figura 4. Prototipo de videolaringoscopio Wi-Mac-Multivision completamente integrado. (mango y hoja Macintosch + base de bicicleta + cámara + PowerBank + smartphone).

excluyeron pacientes quienes se encontraron hemodinámicamente inestables y/o presentaron riesgo de broncoaspiración al momento de la intubación orotraqueal.

En el caso de la evaluación del videolaringoscopio se recolectó la siguiente información en una ficha: éxito en intubación orotraqueal, número de intentos que ameritó, tiempo de intubación, clasificación según Cormack-Lehane, utilización de maniobras externas: B.U.R.P. (Backward, Upward, Rightward Pressure) y complicaciones.

Análisis estadístico: Para analizar los datos se empleo el programa estadístico llamado Epilnfo V3.5.4 y Microsoft Excel 2007.

\section{Resultados}

Al momento de iniciar la investigación el Servicio de Anestesiología y Reanimación del Hospital Central de Maracay solo contaba como dispositivo alternativo para intubación orotraqueal de un fibrobroncoscopio marca $\mathrm{Ambu}^{\circledast}$, la cual debido a las características propias de dicho dispositivo no emite una imagen lo suficientemente nítida, mas sin embargo, logra ser suficiente para lograr las intubaciones orotraqueales en los pacientes en los cuales se ha empleado.

Se aplico, un cuestionario a los miembros del Servicio de Anestesiología y Reanimación del Hospital Central de Maracay, en donde de manera unánime (100\% de la muestra) se consideró necesario incluir nuevos dispositivos para el manejo de la vía aérea, y de la misma manera se aprobó el diseño de un prototipo de videolaringoscopio para la intubación orotraqueal y beneficio de los pacientes de dicho servicio. 

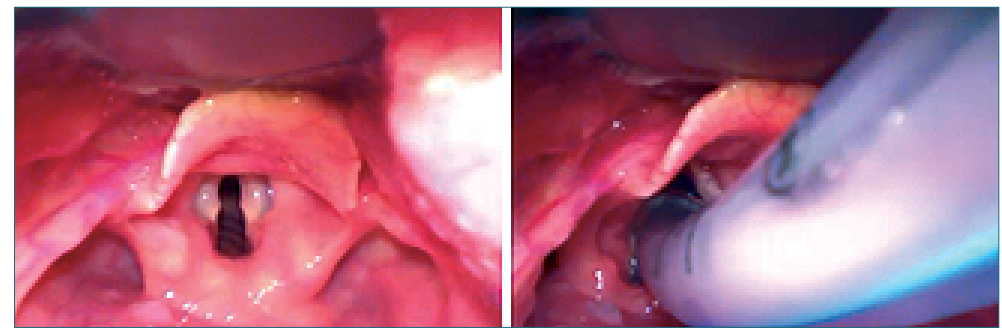

Figuras 5 y $\mathbf{6}$. Imagen obtenida de intubación orotraqueal con VDL Wi-MacMultivision: Visualización de cuerdas vocales y paso del tubo a través de éstas.

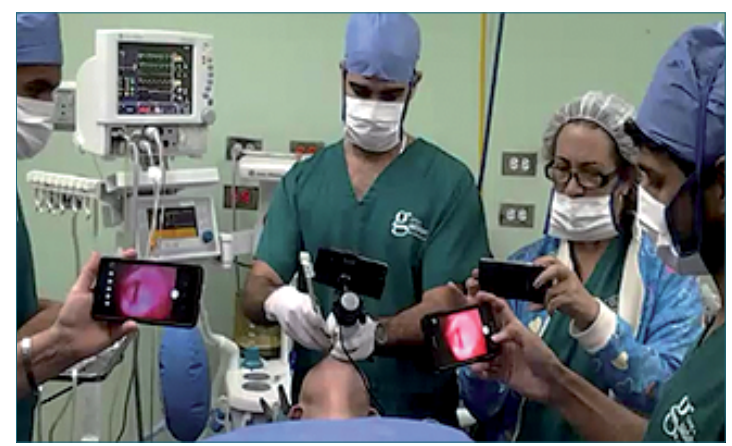

Figura 7. Vista externa de intubación orotraqueal con VDL Wi-Mac-Multivision.

La totalidad de los cuestionados consideró importante desarrollar habilidades en el uso de dispositivos alternativos al laringoscopio tradicional para el manejo de la vía aérea.

Una vez aprobado y realizado el diseño del videolaringoscopio llamado Wi-Mac-Multivision se procedió a aplicar dicho dispositivo en pacientes de dicho servicio para demostrar su efectividad.

El grupo estudiado fue de 32 pacientes los, cuales se clasificaron por género en 18 del sexo femenino $(56,3 \%)$ y 14 del sexo masculino (43,8\%). La edad media de la población estudiada fue de 41,53 \pm 19,30 años. El IMC tuvo una media de 24,25 $\pm 2,61$ $\mathrm{kg} / \mathrm{m}^{2}$. La distribución según la escala de Mallampati fue I (21), II (7), III (3), IV (1).

El $100 \%$ de los pacientes (32) fueron intubados con éxito y de la misma manera ninguno presentó complicaciones al momento del procedimiento, de éstos solo un paciente $(3,1 \%)$ ameritó un segundo intento para lograr la intubación orotraqueal. MackLehane de la imagen obtenida al hacer la laringoscopia con VDL Wi-Mac-Multivision (Tabla 1).

En solo 3 casos (9,4\%) fue necesario el empleo de maniobras externas que mejoraron la visualización de las cuerdas vocales (maniobras de B.U.R.P.) y lograr así la intubación orotraqueal.

El tiempo promedio total en que se logró la intu-

\begin{tabular}{lcc}
\hline & \multicolumn{3}{c}{$\begin{array}{c}\text { Tabla 1. Distribución según la clasificación de } \\
\text { Cormack-Lehane de la imagen obtenida al hacer la } \\
\text { laringoscopia con VDL Wi-Mac-Multivision }\end{array}$} \\
\hline $\begin{array}{c}\text { Clasificación } \\
\text { Cormack- } \\
\text { Lehane }\end{array}$ & Frecuencia & Porcentaje \\
I & 30 & $93,7 \%$ \\
II & 2 & $6,3 \%$ \\
Total & 32 & $100 \%$ \\
\hline
\end{tabular}

bación orotraqueal fue de 29,62 $\pm 7,53$ segundos con el VDL Wi-Mac-Multivision.

\section{Discusión}

A medida que la tecnología y la medicina avanza emergerán nuevas maneras de aplicar y adquirir nuevos conocimientos, tal es el caso de los videolaringoscopios, existen muchos dispositivos que haciendo uso de tecnología de punta facilitan y hacen más seguro el proceso de intubación orotraqueal. Pero la adquisición de dichos dispositivos resulta difícil a países en desarrollo tales como el nuestro, debido al alto costo que estos pueden tener (Airtraq 80 USD, STORZ CMAC 7.980 USD, Glidescope 11.000 USD)[13] y que en algunos casos es necesario adquirir nuevas hojas ya que están diseñadas para un solo uso, o dispositivos que tienen vida útil para un solo paciente.

El Wi-Mac-Multivision tiene un costo total estimado de 45 USD, los artículos empleados para su ensamblaje hacen que sea de mucho más fácil acceso a la hora de disponer de un dispositivo tecnológico, económico, reutilizable para la intubación orotraqueal.

Este prototipo de videolaringoscopio tuvo una tasa de éxito de intubación en un $100 \%$ en los 32 pacientes en los cuales se empleó. En un estudio donde se comparan diferentes tipos de videolaringoscopios vs el laringoscopio Macintosh se evidenció que la tasa 
de intubación exitosa de este último fue de $93 \%$ vs un 100\% de éxito para el C-MAC STORZ vs $97 \%$ Glidescope[16].

En un estudio comparativo realizado en 2014 en Turquía se pudo evaluar bajo simulación en vía aéreas, fáciles y difíciles, la efectividad del C-MAC convencional y el laringoscopio de Macintosh (LM) donde se logró evidenciar en vías aéreas fáciles que con el LM se lograron intubaciones exitosas al primer intento en un $89 \%$, en un segundo intento $11 \%$, en el caso de C-MAC se logró intubar al primer intento en un $96,2 \%$ y en el segundo intento $3,8 \%[17]$. La tasa de éxito al primer intento con el Wi-Mac-Multivision fue de $96,9 \%$ y al segundo intento de $3,1 \%$.

Con respecto a las maniobras empleadas para mejorar la visualización de la glotis y lograr la intubación con el prototipo presentado se necesitó en el $9,4 \%$ de los casos (3). Alper et al.[17], evidenció en su estudio que, utilizando el LM en 26 simulaciones, utilizó dichas maniobras solo en $7,7 \%$, mientras que con el uso de C-MAC (26 simulaciones) no se empleó dicha maniobra en ninguna oportunidad.

Myungju Shin encontró que de las 39 intubaciones realizadas en maniquís de práctica con C-MAC logró una visualización de CL-I en 92,3\% de los casos y CL-II 7,7\%, en el caso de LM logró en 46,15\% una visualización CL-I, 38,46\% CL-II, y 10,26\% y 5,13\% para CL-III y CL-IV respectivamente.

El tiempo de intubación media para el dispositivo propuesto es de 29,62 $\pm 7,53$ segundos. En la intubación descrita por Velázquez-Murillo en México[1] logró la intubación de pacientes con características que los convertían en posibles vías aéreas difíciles utilizando un videolaringoscopio Macintosh artesanal en menos de 120 segundos, en el caso de Grünberg intubó con un videolaringoscopio artesanal Macintosh a un paciente con vía aérea dificultosa prevista en 33 segundos[14].

Hodges et al., compararon en un estudio realizado en 90 pacientes sometidos a cirugías de electivas que ameritaban intubación orotraqueal el dispositivo C-MAC vs LM donde lograron intubaciones en 29,2 \pm 18,6 segundos y $23,5 \pm 9,4$ segundos respectivamente[18].

De la misma manera Myungju Shin et al., lograron intubaciones con el C-MAC en una media de 23,2 segundos y MgGRATH MAC en 21,8 segundos[19].

Jain en 2013, describe intubaciones realizadas por 33 residentes con más de 100 intubaciones orotraqueales cada uno, en este estudio simularon lesión de columna cervical y como dispositivos utilizaron CMAC y LM logrando intubaciones en un tiempo de
$20,21 \pm 7,9$ segundos y $15,55 \pm 2,69$ segundos respectivamente.

Durante la utilización del Wi-Mac-Multivision no se evidenciaron complicaciones, sin embargo, Ruediger et al.[21], en un estudio retrospectivo y comparativo en una Unidad de Terapia Intensiva en 113 (LM) y 117 (C-MAC) pacientes lograron evidenciar 17 y 14 complicaciones respectivamente, de las cuales mencionan lesiones menores a tejidos, sangrado, regurgitación/aspiración, entre otras.

La comparación realizada entre dispositivos en 2011 por McElwain et al., en pacientes con inmovilización cervical menciona únicamente como complicaciones relacionadas al uso de C-MAC y LM laceraciones menores[22].

\section{Conclusiones}

Se demostró la eficacia del prototipo de videolaringoscopio Wi-Mac-Multivision para lograr intubaciones de los pacientes atendidos por el Servicio de Anestesiología y Reanimación, dicho dispositivo surge como una alternativa tecnológica y eficaz para el Hospital Central de Maracay, adicional de esto su bajo costo y fácil manejo lo hace un dispositivo fácil de replicar.

No basta con lo anterior mencionado, dicho dispositivo tendría ventajas sobre el aprendizaje y capacitación del personal con poca o nula experiencia que labore en el Hospital Central de Maracay, como bien se sabe en dicho hospital se forman especialistas en diferentes áreas que habitualmente utilizan laringoscopios tradicionales para la intubación orotraqueal de sus pacientes. De esta manera se estaría brindando mayor seguridad disminuyendo la tasa intubaciones fallidas y las posibles complicaciones que esto conlleva.

Es necesario llegar a un número suficiente de intubaciones para poder lograr un buen grado de entrenamiento, evaluar con más seguridad sus indicaciones, y poder determinar si este sistema puede ser una opción válida para ser usado en países con bajos recursos mientras que no puedan acceder a los videolaringoscopios originales, y de la misma manera evaluar si puede ser empleado en pacientes que por sus características anatómicas son vías aéreas difícil, es por ello que queda una línea de investigación abierta en nuestra institución, la cual se espera pueda continuar.

Dicho dispositivo es la primera versión realizada por los investigadores, se esperan modificaciones en un futuro que hagan más cómoda, práctica y segura su utilización. 


\section{Agradecimientos}

Ante todo a Dios por brindarme la oportunidad de poder vivir mí día a día. Agradecerles a mis padres por el apoyo que desde pequeño me han brindado, mis logros son sus logros sin lugar a duda. A mi esposa quien ha tenido suma importancia en los últimos años para el desarrollo de mis metas. A mi tutor y amigo Miguel Silva por facilitar el desarrollo de mi carrera como anestesiólogo. Y sin lugar a duda a cada uno de los pacientes que me dieron la oportunidad y confianza para aprender a partir de ellos.

\section{Referencias}

1. Guillermo Velázquez-Murillo. Videolaringoscopio Artesanal Macintosh. Anestesia en México. Volumen 28. Número. 2016;1(Enero-Abril):40-5.

2. Apfelbaum JL, Hagberg CA, Caplan RA, Blitt CD, Connis RT, Nickinovich DG, et al.; American Society of Anesthesiologists Task Force on Management of the Difficult Airway. Practice guidelines for management of the difficult airway: an updated report by the American Society of Anesthesiologists Task Force on Management of the Difficult Airway. Anesthesiology. 2013 Feb;118(2):251-70. https://doi.org/10.1097/ ALN.0b013e31827773b2 PMID:23364566

3. Gupta AK, Ommid M, Nengroo S, Naqash I, Mehta A. Predictors of difficult intubation: study in Kashmiri population. Br J Med Pract. 2010 Mar;3(1):307.

4. Vasudevan A, Badhe A. Predictors of difficult intubation: a simple approach. The Internet Journal Of Anesthesiology; 2008;20(2).

5. Griesdale DE, Liu D, McKinney J, Choi PT. Glidescope® videolaryngoscopy versus direct laryngoscopy for endotracheal intubation: a systematic review and meta-analysis. Can J Anaesth. 2012 Jan;59(1):41-52. https:// doi.org/10.1007/s12630-0119620-5 PMID:22042705

6. Martínez $A H$, José MA, Vicente
AM. Tiempo de intubación entre videolaringoscopios: King Vision vs Vivid Trac. Estudio comparativo. ACTA MÉDICA GRUPO ÁNGELES. Volumen. 2016 JulSep;14(3):131-5.

7. Cooper RM, Pacey JA, Bishop MJ, McCluskey SA. Early clinical experience with a new videolaryngoscope (GlideScope) in 728 patients. Can J Anaesth. 2005 Feb;52(2):191-8. https:// doi.org/10.1007/BF03027728 PMID:15684262

8. Ray DC, Billington C, Kearns PK, Kirkbride R, Mackintosh K, Reeve CS, et al. A comparison of McGrath and Macintosh laryngoscopes in novice users: a manikin study. Anaesthesia. 2009 Nov;64(11):1207-10. https://doi.org/10.1111/ j.1365-2044.2009.06061.x PMID:19825056

9. Rothfield K. The video laryngoscopy market: past, present and future. Anesthesiology News Guide to Airway Management. 2014;40(8):29-34.

10. Jungbauer $A$, Schumann $M$, Brunkhorst V, Börgers A, Groeben $\mathrm{H}$. Expected difficult tracheal intubation: a prospective comparison of direct laryngoscopy and video laryngoscopy in 200 patients. Br J Anaesth. 2009 Apr;102(4):546-50. https:// doi.org/10.1093/bja/aep013 PMID:19233881

11. Frerk C, Mitchell VS, McNarry $A F$, Mendonca $C$, Bhagrath $R$, Patel A, et al.; Difficult Airway Society intubation guidelines working group. Difficult Airway Society 2015 guidelines for management of unanticipated difficult intubation in adults. $\mathrm{Br}$ J Anaesth. 2015 Dec;115(6):82748. https://doi.org/10.1093/bja/ aev371 PMID:26556848

12. Howard-Quijano KJ, Huang YM, Matevosian R, Kaplan MB, Steadman RH. Video-assisted instruction improves the success rate for tracheal intubation by novices. Br J Anaesth. 2008 Oct;101(4):568-72. https:// doi.org/10.1093/bja/aen211 PMID:18676418

13. Maya S. Suresh. Role of video laryngoscopes in anesthesia practice. Revista Mexicana de Anestesiología. 2012. Volumen. 2012 Apr-Jun;35 Suplemento 1:S344-61.

14. Grünberg G. Intubación nasotraqueal con "videolaringoscopio artesanal" en paciente con vía aérea dificultosa prevista. Anest Analg Reanim. 2012;25(2):5560.

15. Herrera I. Estudiantes de Anestesiología en el Razetti diseñaron videolaringoscopio artesanal. Consultado: 20-04-2017. Disponible en: http://www.hospitalrazettibarinas.gob.ve/site/contenido/277.html

16. Healy DW, Picton P, Morris M, Turner C. Comparison of the glidescope, CMAC, storz DCI with the Macintosh laryngoscope during simulated difficult laryngoscopy: a manikin study. BMC Anesthesiol. 2012 Jun;12(1):11. https://doi.org/10.1186/1471- 
2253-12-11 PMID:22720884

17. Kılıçaslan A, Topal A, Erol A, Uzun ST. Comparison of the C-MAC D-Blade, Conventional C-MAC, and Macintosh Laryngoscopes in Simulated Easy and Difficult Airways. Turk J Anaesthesiol Reanim. 2014 Aug;42(4):182-9. https://doi. org/10.5152/TJAR.2014.59672 PMID:27366418

18. Hodgetts $V$, Danha RF, Mendonca C, Hillerman C. A randomized Comparison of C-MAC Videolaryngscope versus Macintosh Laryngoscope for Tracheal Intubation. J Anesth Clin Res. 2011;2(09):9. https://doi.org/10.4172/2155-

\subsection{3.}

19. Shin M, Bai SJ, Lee KY, Oh E, Kim HJ. Comparing McGRATH® MAC, C-MAC $®$, and Macintosh Laryngoscopes Operated by Medical Students: A Randomized, Crossover, Manikin Study. BioMed Res Int. 2016;2016:8943931. https:// doi.org/10.1155/2016/8943931 PMID:27703983

20. Divya Jain*, Mandeep Dhankar, Jyotsna Wig y Amit Jain.

Comparación del C-MAC convencional y C-MAC D-blade con laringoscopios directos en simulación de lesión de la columna cervical (estudio en modelo). Rev Bras Anestesiol.
2014;64(4):269-74.

21. Ruediger R. Noppens, Stephanie Geimer, Nicole Eisel, Matthias David and Tim Piepho. Endotracheal intubation using the C-MAC ${ }^{\circledR}$ video laryngoscope or the Macintosh laryngoscope: A prospective, comparative study in the ICU. Crit Care. 2012;16:R103.

22. McElwain J, Laffey JG. Comparison of the C-MAC $®$, Airtraq ${ }^{\circledR}$, and Macintosh laryngoscopes in patients undergoing tracheal intubation with cervical spine immobilization. Br J Anaesth. 2011 Aug;107(2):258-64. https:// doi.org/10.1093/bja/aer099 PMID:21586444 